\title{
Frauenärzte online \\ Klassische Marketing-Strategien ins Internet übertragen
}

$\mathrm{M}$

it der Übertragung klassischer Marketing-Strategien auf das Medium Internet lässt sich immer noch Geld verdienen. Ein aktuelles Beispiel: Für den Berufsverband der Frauenärzte e.V. (BvF) und die Deutsche Gesellschaft für Gynäkologie und Geburtshilfe (DGGG) betreibt die Münchner Firma Sean Monks Ärzte-im-Netz GmbH seit September 2006 die Website www.frauen aerzte-im-netz.de. Kern des Angebotes sind Informationen zu etwa 70 Frauenkrankheiten. Zusätzlich gibt es Fakten und Hintergründe zu Themen wie Krebsfrüherkennung, Impfungen oder Osteoporose. Grafiken und Videoclips mit 3-D-Animationen sollen komplexe medizinische Zusammenhänge veranschaulichen.

\section{Seriöse Informationen}

\section{für Patienten?}

Das Portal richtet sich in erster Linie an Patientinnen, Angehörige sowie medizinisch interessierte. Ziel der Website: „Im Internet kursieren sehr viele falsche und strittige Informationen zu verschiedensten Themen und Erkrankungen der Frau. Die zwei wichtigsten Organisationen im Bereich der Frauenheilkunde und Geburtshilfe in Deutschland bieten nun eine umfassende Internetplattform mit seriösen Informationen für Patienten an. Die Inhalte in diesem Portal entsprechen den medizinischen Leitlinien und basieren somit auf den neuesten wissenschaftlichen Erkenntnissen“, betont Dr. Christian Albring, Präsident des Berufsverbandes der Frauenärzte und Beisitzer im Vorstand der Deutschen Gesellschaft für Gynäkologie und Geburtshilfe beim DGGG-Jahreskongress im September 2006 in Berlin. Man habe dabei vor allem großen Wert auf die Verständlichkeit der Erläuterungen und ein werbefreies Angebot gelegt. Ergänzt wird das Portal mit interaktiven Angeboten, etwa einem Zykluskalender zum Herunterladen sowie einer Erinne-

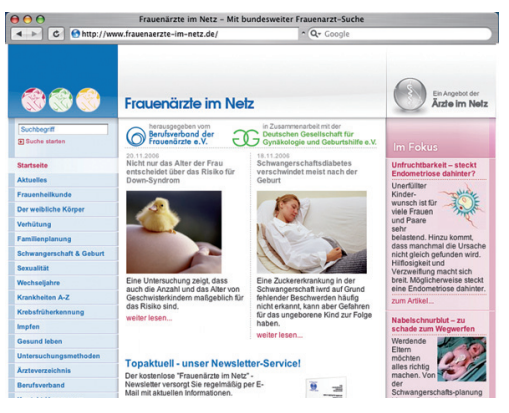

rungsfunktion für regelmäßige Krebsfrüherkennungsuntersuchungen. Zudem gibt es ein bundesweites Ärzte- und Klinikverzeichnis mit Suchfunktion.

Seite ist „werbefrei“ - finden die Initiatoren

Die vorgestellte Website ist Teil der Marketingstrategie der beteiligten Verbände, soll also Akzeptanz und Nutzung frauenärztlicher Leistungen verbessern. Damit ist sie natürlich nicht „werbefrei“, wie die Initiatoren finden. Aufmerksamen Lesern wird dies spätestens angesichts der grundsätzlich positiv gestimmten Bewertung der hormonellen Kontrazeption oder den „Erfolgen“ der modernen Sterilitätsmedizin deutlich. Hinsichtlich des letzteren Themas heißt es zudem im Stil des tausendjährigen Reichs: „Auf Grund der schlechteren Überlebenschancen aller Feten einer Mehrlingsschwangerschaft kann der Mutter in einem frühen Entwicklungsstadium die selektive Tötung (Fetozid) desjenigen Embryos mit den geringsten Überlebenschancen angeboten werden“ (Zitat online seit 18.9.2006). Dass dieser selektive Fetozid regelmäßige Begleiterscheinung der modernen medizinischen Kinderlosigkeits-„Therapie“ ist, wird - wie nicht anders zu erwarten - verschwiegen. Aus kollegialer Sicht ist zu hoffen, dass solche Ausführungen nicht die Auffassung aller Frauenärztinnen und Frauenärzte ausdrücken.

Rainer H. Bubenzer, Hamburg 